\title{
Machine Learning Techniques of Contact Aware Communication System:
}

\section{A Comprehensive Overview}

Mohsin Shahzad ${ }^{1, *}$, Kashif Hussain Memon ${ }^{2}$, M. Ali Qureshi ${ }^{2}$ and Fareeha Zahoor ${ }^{3}$

\author{
${ }^{1}$ Department of Computer Engineering, UET, Taxila 47050, Pakistan \\ ${ }^{2}$ University College of Engineering and Technology, IUB, Bahawalpur 63100, Pakistan \\ ${ }^{3}$ Department of Computer Science \& IT, IUB, Bahawalpur 63100, Pakistan
}

11cp40@uettaxila.edu.pk

\begin{abstract}
Machine Learning (ML) and Artificial Intelligence(AI) have revolutionized almost all fields that are linked to the acquisition of intelligent behavior in the real world. It is an attractive alternative for a researcher of artificial intelligence. Contrary to rule-based programming, ML is an algorithmic approach in which learning comes from existing data. The more data we have these computer systems look at, we say we're 'training' the computer system, and as the computers begin to identify patterns in the data, identify abnormalities in the data from these abnormalities we improve the system architect according to the requirement. This article introduces the use of comprehensive concepts of machine learning, in general, particular, and their potential applications in communications. Furthermore, the current state and futuristic potentials of enabling universal communication with implications of machine learning methods have been explained. In this review paper, we offer a comprehensive talk on distinctive methods/techniques of information analytics, artificial intelligence (AI), and machine learning (ML) moved forward the contact aware communication system.
\end{abstract}

Keywords: Machine Learning, Next Generation, Contact Aware, Communication System, and Machine-type Communications.

\section{Introduction}

The fifth-generation wireless network plays an important role in worldwide communication and plays an essential role for the future connected society which is expected to connect billions of devices to enable worldwide communication in the future. As the network size increases the complexity of network base stations, user equipment also increases. New challenges are arising for denser and complex network planning [1].

Architecture requirements for $5 \mathrm{G}$ and surpassing will be predictably heterogeneous and multi-tire with the ultra-dense implementation of small cells to acquire cost-effectiveness and increase capacity. The futuristic wireless network has to responsible for formulating, construct, and invigorating potentials based on the practical posture through the tight systematization among different nodes, tiers and intercommunication layers. These challenges highlight that existing network design strategies, which utilize a fairly simple statistics experience, delivers unacceptable performance [2].

The exponentially developing number of gadgets for machine-type communication (MTC) adds to the difficulty of this ultra-dense arrangement and empowers communication across the globe. To realize high association, truth and stability, incredibly fast travel times and moo idleness [3], cutting-edge MTC applications enabled by $5 \mathrm{G}$ and past would require simple remote systems. The strongest need is always for nonstop and healthy service. For some occasions, taking an MTC application offline will trigger crucial trade misfortune or inadequate customer encounters, and many of the MTC gadgets are resource-constrained and would not be able to rely entirely on their claim-limited assets to meet their handling requests [4]. These idleness-specific applications cannot be transferred to the structured controller due to cloud latency, transmission space, or another hang-up. The data sets produced from these gadgets will be amazingly assorted and may have incorrect values on a large scale [5].

* Corresponding author. Tel.: +92-62-9250338

E-mail address: $11 \mathrm{cp} 40 @$ uettaxila.edu.pk 


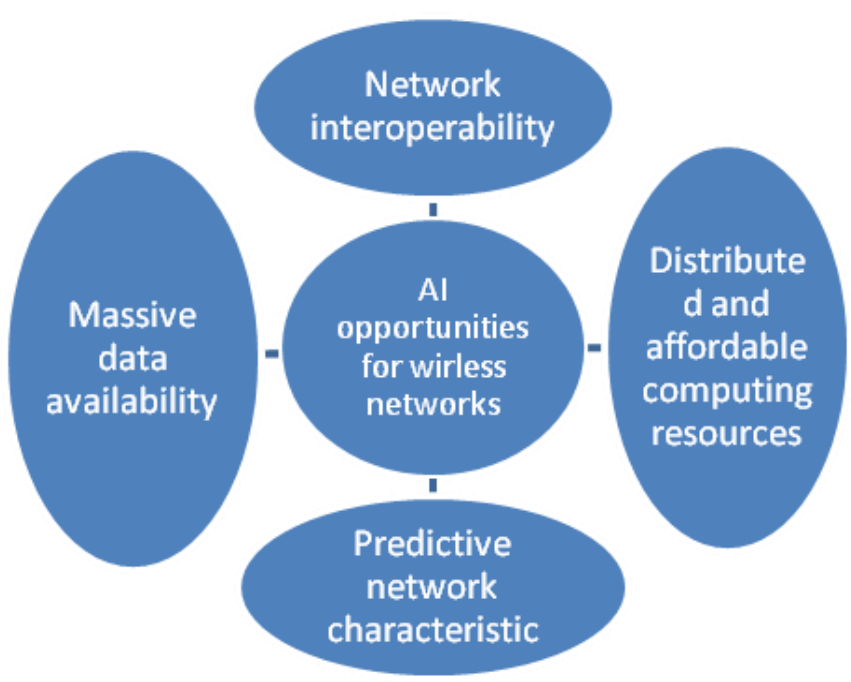

Fig. 1. Encouraging conditions for the endorsement of machine intelligence techniques in the nextgeneration wireless networks.

A variety of unused knowledge-hungry MTC immersive use-cases will appear counting wearables, simulated substances, and brilliantly item and back- frameworks, where most of them will leverage a built-in back-end information base to supply context-aware administrations [5] [13]. All these compel the following era coordinate to adopt a brilliantly and context-aware method for organizing, schedule, review, and extension. The swift revolt of semiconductor firms and creativity in computation was powered by the starting point of a shrewd moment. This is seen by the tight integration of organized data frameworks, gadgets for detection and communication, sources of data, choice-making, and cyberphysical foundations. The small remote sensor, smartphone, shrewd gadgets learning from previous encounters, and data set are the strong witnesses of the advancement of semiconductor companies that make gadgets smaller and shrewdly. These promising changes encourage transmitted computing resources not just within the cloud, but also within the hubs of mist and edge. Both haze and edge computing attempt to bring the insights and capabilities of handling closer to where the data begins.

\subsection{Edge computing and Fog Computing}

Edge computing streamlines the operation stream from the Internet of Things (IoT) gadgets and offers an investigation of real-time results. Edge computing allows IoT gadgets provided with information to be prepared closer to where it is made rather than sending it to information centers or clouds over long courses. Doing this computation closer to the edge of the enterprise helps organizations to examine critical information over various firms, counting retail, well-being care, broadcast communications and back in near real-time a need of organizations.

Fog computing or misting may be clustered computing in which data and software are shared between sources of information and the cloud. Mist computing takes the cloud's points of interest and power closer to where data is generated and operated upon. The aim of fogging is to improve efficiency and reduce the volume of data for handling, analysis, and power transferred to the cloud.

To promote investigation, the beautiful edge center decides which details blunt data placed away locally and which information can be forwarded to the cloud. All the rudimentary input information will initially be modified to the required network convention (such as HTTP) inside haze computing, some time recently sent to the mist hubs. In this way, higher-level data content is prepared, deleted and submitted to the cloud to facilitate analysis inside the mist gadgets (for illustration, shrewdly switches, get to focuses, IoT doors). In this way, the organization allowed by edge and haze enables disseminated computation, power, control, communication and organization capabilities by reducing the transmitted information and cloud workload, inactivity and system reaction time, particularly for applications 
requiring localized and location-dependent data[6]. The hubs, sensors and MTC devices can learn from past data and states and predict the required measurement and design organization. All these opportunities enable efficient and adaptable allocation and administration of assets, convention stack arrangement, signaling method and optimization of physical layers, and encourage existing gadgets to tackle the capabilities of sensors, edge, haze and cloud-based computing stages, and motors for information analytics[7]-[9]. Besides, these provide optimal conditions for creating a strongly organized remote organization by adopting AI principles (see Fig. 1) by consolidating research, reasoning and decision-making resources that are essential for the capacity to realize contextawareness. The use of AI standards at distinctive hubs is commonplace after generation (see Fig. 2). This paper provides a systematic use of machine learning in all-inclusive communication and different techniques for upgrading all-inclusive communication using AI strategies.

\section{Information Learning and Acknowledgement of Information}

Efficient information learning and acknowledgment of information are the future requirements of the network it helps to realize situation awareness and optimized decisions (see Fig. 3). There are lot of challenges in data acquisition like IoT [5].

AI-based tools based on machine learning algorithm in which our system learn from the previous and current input data and different levels could be applied for knowledge extraction [10]. This includes the cell level, cell cluster level and user level for knowledge extraction. There are different sources to collect information these sources will be user and external devices. The user data provides the user description information and the external specific information that is obtained from the behavior of different sources such as network, sensor and channel measurements [11].

There is the possibility that the same content will be requested by different users with different quality. For example, one user requested video content and same time another user request the same video content but with a different quality to meet this requirement the edge devices apply adaptive caching techniques based on the quality of service (QoS) requirement [12].

We can also enable devices to create multicasting opportunities for certain content via coded multicast transmissions by enabling the coded cache [13]. When we are collecting data, a given edge may collect data from different sources with different connectivity criteria [14]. It is important to get effective knowledge on the best network exploitation and management.

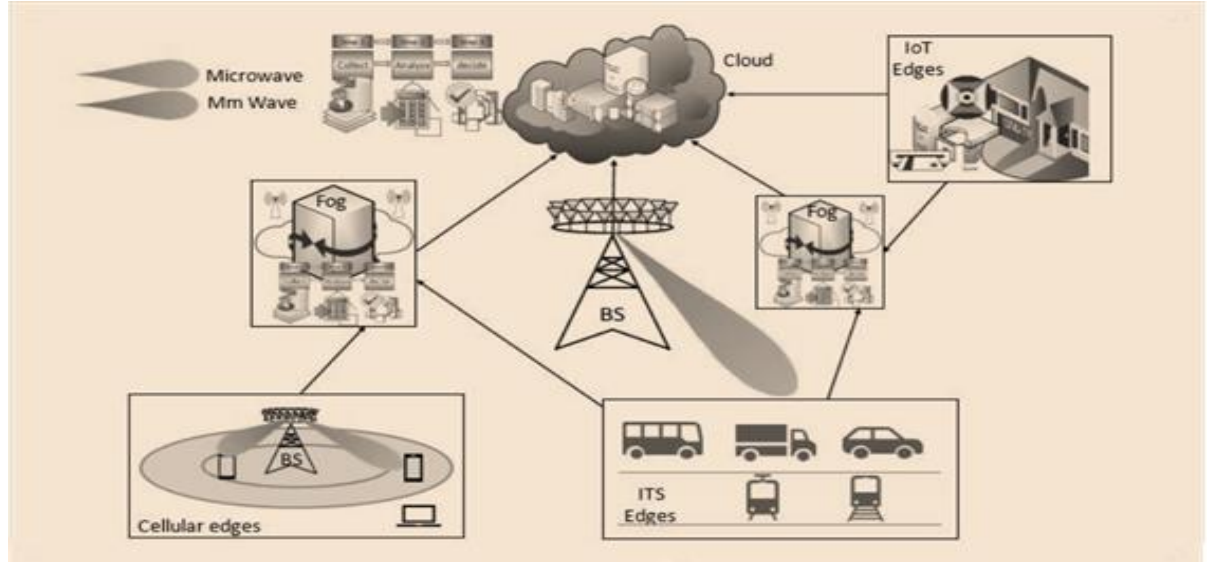

Fig. 2. Typical next-generation network with AI principles; Learning, reasoning and decision making.

Noble learning strategies on the network may be used, such as machine learning or deep learning profits on the network, which is very important for building a knowledge base. In general, it is any information that can be used to describe the environment, the environment, identity, preferences, and activities. The context can be linked to complex system operations at different levels, from the actual machine level to the application level [15]. Insufficient data analysis (semantically) makes it easy for wireless 
operators to optimize their network traffic. However, to make the content of the network understandable, you need to understand the content of the signal. One of the ways to understand the content of the data is to create semantic-aware ontology using the predictive vocabulary of terms and concepts [16]. Existential knowledge may have a variety of cohesive abilities to identify a few fields, attributes, and relationships. In this regard, the developers of [16] propose the case of MyOntoSens, the proof of browser knowledge, and the online type 2 dialect database of the Module dialect. Using prote'ge 'and pre-validated with pellet contemplated, the planned KB was introduced. A theory for remote sensor systems (WSNs) devoted to the interpretation of sensor highlights and awareness was demonstrated in a comparable environment in[17][18].

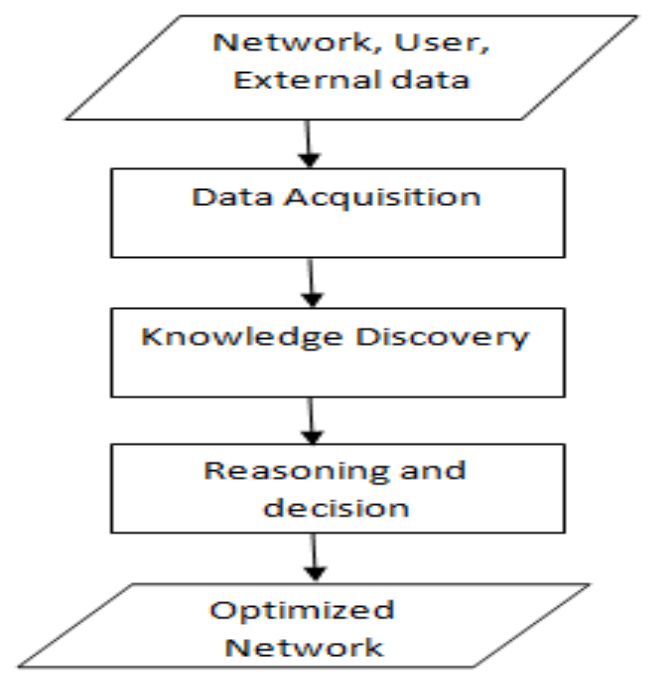

Fig. 3. Optimized network design with AI techniques.

Contextual understanding also helps produce context-sensitive information (summary) that will use fewer wireless resources to convey. For example, if the base station wants to send text information to a user, it can only transmit its encrypted data. The user will then extract only desired content from the context by using appropriate encryption techniques and large data technologies. Because the context is different from the user's knowledge about the content, the encryption and decryption technology may vary between users [15]. There are two abstractive and extractive approaches that are generally adopted for content summarizing. In the extractive approach when the original information is approached at the user end that point that information is decoded and only required content are used from this original information on the other hand as the abstractive approach may use new words as part of the summary information [19] [20]. While most current methods can generate important conclusions, they are far from being able to generate an enlightening summary. One of the main reasons for this is peaceful unions and the exchange of unpublished information. It is difficult to extract urgent and relevant information in the context of translations. Making form formulas is part of the research work [19].

\section{$3 \quad$ Networking Planning}

The performance of a next-generation is depending on the latest techniques of emerging technology in networks. This technology depends on different techniques, likely frequency, number of bits, distance, coding scheme and network energy usages in both cases idle (data not transfer between a base station and user) and active (data transfer between a base station and user). A well-planned and well-managed network must be guaranteed the satisfaction of the user in terms of the reliability, latency and data rate collection of these terms called QoS and the network operator/company requirements based on cost. The machine learning techniques are the best opportunity for the planning of different parts of the communication by classifies the available data of the network and user. 


\subsection{Energy consumption, Node deployment and RF planning}

Remote systems of the next generation require high throughput and reliability, which are extremely dynamic, likely prepared to protect the vast number of customers with the most reduced transmission power with moving and flying BSs, and highlighted without any misfortune of optimum conditions[21]. Because of the current arrangement preparation methods, which are practically inactive and illustrated inexpensive field studies, they are not appropriate for remote schemes of another generation[2][22].

Some machine vision, discourse recognition and mechanical autonomy are utilized for AI techniques. But less use of AI techniques has recently received interest in the group inquiry within the coordinated planning sector. For instance, for substance popularity, a machine learning approach is suggested and ask for delivery predictions of a cache where the strategy inside the enterprise considers a few variables counting cache structure and update process that is determined by using the substance request distribution and frequency of the customer, and flexibility design[23].

In[24] the highest Internet speed is expected to arrange for domestic customers from the center. An AIbased framework with graph hypothesis-based problem descriptions assists us in suggesting the arrangement of structures to robotize the system. Mixed-integer linear programming (MILP), ant colony optimization (ACO), and genetic algorithm (GA) were related to illuminating the problems. Modern cell, radio recurrence (RF) and spectrum of the $5 \mathrm{G}$ remote arrangement incorporate the use of AI requirements for radio get to arrange (RAN) arrangement.

The analysis is carried out by the preparation of input evidence from multiple sources, through learningbased grouping, assumption and clustering models, and the extraction of substantial knowledge to drive the choices provided by the Child-based 5G arrangement. Remote systems contribute to a growing share of the data transmission invention (ICT) platform in the optimization of vitality. RANs primarily use the overall 70 percent control of a remote network, particularly within the BS, as the BS arrangement base for crest operation loads is now designed, and for the most part, remains dynamic independent of the monumental varieties in the activity stack[25]. For the long-term shrewd citation, this promotes the green ICT. In the next generation of remote systems, different AI techniques were suggested to empower less control architecture. $\operatorname{In}[26][27]$, the author stressed the reduction of the vitality use of personal gadgets servers, network hardware, air conditioners, etc. in the strategy of customary green computing of the full structured systems by managing each part individually depending on real network device usage.

\subsection{Configuration parameter and service planning}

When moving from one more seasoned generation to the next higher generation, network components, as well as setup parameters, must be changed. These parameters are between 1000 and 1500, individually, for occasions in regular $3 \mathrm{G}$ and $4 \mathrm{G}$ hubs. This pattern is predicted to persist and the $5 \mathrm{G}$ arrangement hub recommended at a late date will have 2000 or more parameters. The other generation network will continuously generate unused highlights (movable, high speed, less use of vitality, etc.) in extension, divergent from the present and past generation networks that deliver inactive administrations, and set up sufficient resource arrangement and provisioning procedures while maintaining nimbleness and strength of circumstances.

These include conditions for another age network to acquire parameter varieties, learn vulnerabilities, plan parameters of the network, anticipate rapid and potential problems, and provide timely arrangements by environmental collaboration[25]. For convention stack configuration, signaling method, and physical layer method optimizations, the use of huge information analytics techniques has been addressed in [9]. Future cities need well-organized wired and distributed networks with an uninterruptible web network and the ability to manage information in an adaptable, real-time and decentralized manner. One way to overcome this problem will be to relay fog-based remote engineering to the AI coordinates that enable the arrangement to manage information using a variety of disseminated hubs. This will include assistance for coordinating the status review, separating planned problems and organizing modern hub sending using AI strategies[1]. 


\section{Network Operation and Management}

In each network service and management, energy and bandwidth proficiency, idleness, stability, and safety are the most critical requirements. Satisfactory efficiency for both service providers and endusers relies on maximizing these parameters. Furthermore, various AI strategies have been suggested based on simple and real-time learning and decision-making algorithms that usually incorporate these optimization parameters.

\subsection{Resource allocation and management}

For intelligent routing, asset selection, management and optimization of remote systems[22], distinctive AI approaches such as deep learning have been organized. The ability of AI to understand the channel allocation problem in remote communication is considered in [28]. The AI-based approach is better implemented than that of heuristic and genetic randomized methods.

\subsection{Security and privacy protection}

Security is the most critical aspect of remote transmission, counting listening and holding in as waves travel unreservedly in circumstances, helpless to malicious attacks. Security and security assurance are also essential aspects of today's remote communication infrastructure. Communication system apply security using different techniques like encryptions, encoding and many other protocols stack on different layers. Significant utilization of AI techniques can be beneficial to getting wireless security. In [30] author proposed a malicious attack detection plot using an irregular main dissemination-based artificial immune system (AIS).

\subsection{Latency optimization}

Next-generation wireless networks are the most important issue is latency optimization. New technology is based on the static and moveable structures due to environment constrain some delays have occurred. The distinctive aspect of automation for vehicle systems has been defined by the USA transport office, extending from simple driver assistance (level 1) to full computerization mode (level 5). In reality, as of late, it has been shown by an effort to advance human decision-making precision by using natural language generation techniques from loose knowledge. By modeling questionable terms such as tiny and big, which are norms in lifestyle conversation, using fuzzy logic theory[31], such a phonetic representation of knowledge may well be planned. By using historical data, machine learning techniques may be used to answer these tests. The estimation of computational prerequisites helps the gadgets to schedule the computational capital in advance to minimize global inactivity[29]. A quick summary of the distinctive problems within the remote AI-enabled arrangement is shown in Table 1.Table 1. Main issues in Communication System based on AI.

\begin{tabular}{cll}
\hline Data acquisition and knowledge discovery & \multicolumn{1}{c}{ Ref. } \\
\hline$\bullet$ & Coded (adaptive) caching & \\
- & Implementation of semantic-aware philosophy from & {$[12],[13]$} \\
& network data & {$[16]-[18]$} \\
- & The robust revelation of details from unreliable & {$[5]$} \\
& (lost) knowledge & \\
\hline Network planning & {$[10],[24]$} \\
\hline$\bullet$ & Node arrangement and allocation for radio & {$[25],[26]$} \\
& frequency & {$[29]$} \\
- & Energy consumption modeling and prediction & {$[1],[9]$} \\
\hline
\end{tabular}


- Caching and computing placement and content update

- Method for parameters and operation setup

\begin{tabular}{|c|c|}
\hline Vetwork operation and management & \\
\hline $\begin{array}{l}\text { - Channel selection } \\
\text { - Security: Spoofing attack } \\
\text { - Latency: Context-aware edge computing and } \\
\text { scheduling }\end{array}$ & $\begin{array}{l}{[28]} \\
{[30]} \\
{[5],[29]}\end{array}$ \\
\hline
\end{tabular}

\section{$5 \quad$ Discussion \& Conclusion}

Design the wireless network using traditional way use power consumption and the system is very complex. The planning network was able to make the most excellent use of the available resources, successfully using Machine Learning for the case, power, spectrum, as well as basic frameworks and optimization. Machine Learning utilizes the methods of constructive, self-aware, self-adaptive, and prescient networking as needed [32]. Huge quantities of past information were required by the planning network.

Next-generation wireless networks that are more efficient and complex with access to business and information technologies can lead to several digital challenges in network management, management and navigation. Wireless data, however, may come from a variety of sources, including information systems, sensors and communication devices. Additionally, new cloud computing structures that target distribution, computer, networking, communications, and network performance will be provided to leading users of wireless platforms. This review paper gives a comprehensive overview of the utilization of AI joining machine learning, information analytics, and NLP strategies for improving the proficiency of communication systems. We have given a comprehensive overview of the utilization of these techniques for productive remote information securing and information discovery, planning, operation, and administration of next-generation communication systems.

\section{References}

1. Mirza Golam Kibria, Kien Nguyen: Big Data Analytics, Machine Learning, and Artificial Intelligence in Next-Generation Wireless Networks. IEEE Access, Volume: 6,

pp. 3232832338 (2018).

2. A. Munir, P. Kansakar, and S.U. Khan: IFCIoT: integrated Fog Cloud IoT: A novel architectural paradigm for the future internet of things. IEEE Consumer Electronics Magazine, vol. 6, no. 3, pp. 74-82 (2017).

3. T.E. Bogale and L. Le: Massive MIMO and mmWave for $5 \mathrm{G}$ wireless HetNet: Potential benefits and challenges. IEEE Vehic. Techno. Mag., vol. 11, no. 1, pp. 64-75 (2016).

4. M. Weiner, M. Jorgovanovic, A. Sahai, and B. Nikoli: Design of a low-latency, a high-reliability wireless communication system for control applications. in Proc. IEEE Int. Conf. Commun. (ICC), pp. 3829-3835 (2014).

5. M. Chiang and T. Zhang: Fog and IoT: An overview of research opportunities. IEEE Internet of Things, vol. 3, no. 6, pp. 854-864 (2016).

6. X. Wang and Y. He: Learning from uncertainty for big data: Future analytical challenges and strategies. IEEE Systems, Man, and Cybernetics Magazine, vol. 2, no. 2, pp. 26-31 (2016).

7. Ruilong Deng, Rongxing Lu, Chengzhe Lai, Tom H Luan, and Hao Liang: Optimal workload allocation in fog-cloud computing toward balanced delay and power consumption. IEEE Internet of Things Journal, vol. 3, no. 6, pp. 1171-1181 (2016).

8. R. Bendadouche, C. Roussey, G. De Sousa, J. Chanet, and K-M. Hou: Extension of the semantic sensor network ontology for wireless sensor networks: The stimulus-WSN node- communication pattern. in Proc. 5th Int. Conf. Semantic Sensor Networks, Vol. 904, pp. 49-64 (2012). 
9. M. Compton, A. A. Henson, L. Lefort, H. Neuhaus, and A. P. Sheth: A survey of the semantic specification of sensors. Proc. CEUR Workshop, pp. 17-32 (2009).

10. E. Cambria and B. White: Jumping NLP curves: A review of natural language processing research. IEEE Comp. Intelligence Mag., pp. 48-57 (2014).

11. T. Hirao, M. Nishino, Y. Yoshida, J. Suzuki, N. Yasuda, and M. Nagata: Summarizing a document by trimming the discourse tree. IEEE/ACM Trans. Audio, Speech, and Lang. Process, vol. 23, no. 11, pp. 2081-2092 (2015).

12. Y. Fadlallah, A. M. Tulino, D. Barone, G. Vettigli, J. Llorca, and J.M. Gorce: Coding for caching in 5G networks. IEEE Commun. Magazine, vol. 55, no. 2, pp. 106-113 (2017).

13. D. Ohmann, A. Awada, I. Viering, M. Simsek, and G.P. Fettweis: Achieving high availability in wireless networks by inter-frequency multi-connectivity. IEEE International Conference on Communications (ICC), pp. 1-7 (2016).

14. F. Chiti, R. Fantacci, M. Loreti, and R. Pugliese: Context-aware wireless mobile autonomic computing and communications: Research trends and emerging applications. IEEE Wireless Commun., vol. 23, no. 2, pp. 86-92 (2016).

15. M. Alzenad, A. El-Keyi, F. Lagum, and H. Yanikomeroglu: 3-D placement of an unmanned aerial vehicle base station (UAV-BS) for energy-efficient maximal coverage. IEEE Wireless Communications Letters, vol. 6, no. 4, pp. 434-437 (2017).

16. M. Chiang, S. Ha, C. L. I, F. Risso, and T. Zhang: Clarifying Fog computing and networking: 10 questions and answers. IEEE Communications Magazine, vol. 55, no. 4, pp. 18-20 (2017).

17. Y. Sun, H. Song, A. J. Jara, and R. Bie: Internet of things and big data analytics for smart and connected communities. IEEE Access, vol. 4, pp. 766-773 (2016).

18. S. Han, C. L. I, G. Li, S. Wang, and Q. Sun: Big data-enabled mobile network design for 5 G and beyond. IEEE Communications Magazine, vol. PP, no. 99, pp. 2-9 (2017).

19. J. Prez-Romero, O. Sallent, R. Ferris, and R. Agust: Knowledge-based 5G radio access network planning and optimization. International Symposium on Wireless Communication Systems (ISWCS), pp. 359-365 (2016).

20. J. Prez-Romero, O. Sallent, R. Ferris, and R. Agust: Artificial intelligence-based 5G network capacity planning and operation. International Symposium on Wireless Communication Systems (ISWCS), pp. 246-250 (2015).

21. G. Paschos, E. Bastug, I. Land, G. Caire, and M. Debbah: Wireless caching: Technical misconceptions and business barriers. IEEE Communications Magazine, vol. 54, no. 8, pp. 16-22 (2016).

22. L. Nachabe, M. Girod-Genet, and B. El Hassan: The unified data model for wireless sensor network. IEEE Sensors Journal, vol. 15, no. 7, pp. 3657-3667 (2015). 